\title{
Tai Chi, Qigong, and the Treatment of Hypertension
}

\author{
Robert W McGee* \\ Department of Graduate and Professional Studies in Business, Fayetteville State University, USA \\ *Corresponding author: Robert W McGee, Department of Graduate and Professional Studies in Business, Fayetteville \\ State University, USA
}

\section{ARTICLE INFO}

Received: 㭡 September 15, 2021

Published: 㓞 September 30, 2021

Citation: Robert W McGee. Tai Chi, Qigong, and the Treatment of Hypertension. Biomed J Sci \& Tech Res 39(1)-2021. BJSTR. MS.ID.006256.

\begin{abstract}
Qigong and tai chi are both considered tools in the toolbox of traditional Chinese medicine (TCM). Qigong has been in existence for thousands of years, whereas tai chi is of a more recent origin. Both are considered low-impact exercises that have numerous health benefits. The present article reviews some studies on the treatment of hypertension and high blood pressure using one or both of these tools of TCM. Numerous studies have found that tai chi and qigong exercises can have a significant positive impact on blood pressure, nitric oxide and endothelin-1 levels.
\end{abstract}

Keywords: Qigong; Chi Gong; Tai Chi; Taiji; Taijiquan; Traditional Chinese Medicine; TCM; Hypertension; Blood Pressure; Nitric Oxide; Endothelin-1

\section{Introduction}

Tai chi, also referred to as taiji or taijiquan, is considered both a martial art and a kind of low-impact exercise. Its origins are unclear, but it apparently dates back at least to the thirteenth century. The oldest style is the Chen style, which originated in the Chen village in China [1,2]. The second oldest style, and also the most popular style, practiced by more people than any other style, is the Yang style [3]. The other main styles are the $\mathrm{Wu}$ and $\mathrm{Wu}$ Hao styles [4], and the Sun style [5], which is the youngest of the five main styles. The various styles of tai chi have much in common, although there are some differences, which we need not discuss in this article. One of the main common features of all styles of tai chi is that they generate healing life energy (qi, pronounced chee), which serves to boost the body's immune system and prevent the onset of illness and disease. Qi energy also has a beneficial effect on treating existing illness. Many articles and books have been written about the health benefits of tai chi [6-7], so we need not go into the details here. Suffice it to say that many medical studies have found that the regular practice of tai chi can lead to many health benefits, including the treatment of existing diseases and illnesses.

Qigong has been around a lot longer than tai chi, perhaps thousands of years [8]. Many books and articles have been written about this traditional Chinese medicine tool as well [9-74]. It is also a set of gentle exercises that generate qi, which has beneficial healing effects for a wide variety of ailments, including, but not limited to ankylosing spondylitis [75-76], anxiety and stress reduction [77-82], arthritis [83-89], autism [90], back pain [91-92], cancer [93-115], cognitive impairment [116-119], COPD [120-121], COVID-19 [122-123], depression [124-134], elder care [135-138], fibromyalgia [139-141], longevity [142-144], Parkinson's Disease [145-146], and traumatic brain injury [147], to name a few. The present article focuses on the beneficial effects of tai chi and qigong exercises on hypertension and blood pressure. It reviews a few studies that have found beneficial effects and cites a number of other studies for further reading and research.

\section{Methodology}

The PubMed.gov database [148] was searched to find studies that had been done to determine the effectiveness of tai chi and qigong exercises on blood pressure and hypertension.

\section{Findings}

The findings reported upon in this article are representative of the numerous studies that have been done examining the effects of 
tai chi and qigong on blood pressure and hypertension. Additional studies on this topic are cited in the reference section below Liu et al. [149] conducted a meta-analysis to determine the effectiveness of tai chi and qigong exercises in the treatment of essential hypertension (EH). Specifically, they looked at blood pressure (BP), levels of nitric oxide (NO), and endothelin-1 (ET-1). Exercises were performed from 1.5 to 6 months. Nine randomized controlled tests (RCTs) of $516 \mathrm{EH}$ patients in China found that those who did the exercises were able to reduce both systolic and diastolic blood pressure. The exercises also contributed to higher NO blood levels and lower ET-1 blood levels. Although the difference in treatment outcomes using tai chi and qigong exercises versus antihypertensive drugs was statistically insignificant, combining the two therapies resulted in significantly better outcomes than what would occur using only tai chi and qigong or drug therapy. Thus, tai chi and qigong exercises were equally effective as drug therapy in the treatment of hypertension, only without the side-effects that may be present with drug therapy. Liu et al. concluded that tai chi and qigong exercises could be an effective complementary and alternative therapy for $\mathrm{EH}$ patients.

The tai chi exercises varied by study, and included the Yang24 form, Yang-8, and Chen-style tai chi. The qigong exercises also varied by study, and included Mawangdui Daoyinshu and Baduanjin, among others. Subgroup analyses were performed for the different types of tai chi and qigong, and some were found to be more effective than others. One subgroup analysis of changes in systolic blood pressure ranked the effectiveness of the various exercises as follows, from most to least effective:
a) Chen-style tai chi
b) Mawangdui Daoyinshu Qigong
c) Self-compiled qigong
d) Yang-style tai chi

An examination of different subgroups found that some tai chi and qigong exercises were more effective than others in lowering diastolic blood pressure. The ranking, from most to least effective, was:
a. Chen-style tai chi
b. Self-compiled qigong
c. Mawangdui Daoyinshu Qigong
d. Yang-style tai chi

Liu et al. concluded that Chen-style tai chi might be most effective in reducing blood pressure, while Yang-style tai chi might be the least effective. The authors also compared the effectiveness of the various tai chi and qigong exercises on improving NO levels. The ranking from most to least effective was:
a. Yang-style tai chi
b. Baduanjin Qigong
c. Mawangdui Daoyinshu Qigong

Chen-style tai chi and self-compiled qigong were not statistically significant in improving NO levels. The authors also analyzed subgroup data on the effectiveness of tai chi and qigong in reducing ET-1. The ranking from most to least effective was:
a. Baduanjin Qigong
b. Yang-style tai chi
c. Mawangdui Daoyinshu Qigong

Self-compiled qigong was found not to be statistically significant in lowering ET-1 levels. Thus, it appears that Baduanjin and Yangstyle tai chi may be more effective than other exercises in improving NO and ET-1 scores.

If one were to interpret the findings of this study, one might conclude that choosing qigong and or tai chi therapy might be superior to drug therapy for the treatment of EH for two reasons. Although the study found that qigong/tai chi therapy and drug therapy are equally effective in treating EH, qigong/tai chi therapy has two distinct advantages over drug therapy: qigong/tai chi therapy has no adverse side-effects, and it does not cost anything. Drug therapy, on the other hand, sometimes has adverse sideeffects, and it is not free. The study also found that combining qigong/tai chi therapy with drugs might be superior to choosing just one of the two options.

Pan et al. [150] conducted a systematic review of randomized controlled trials on the effects of tai chi on blood pressure, body mass index (BMI), and quality of life (QOL) on patients suffering from hypertension. Their meta-analysis of 24 studies containing 2,095 patients $(1,074$ in the treatment group and 1,021 in the control group) found that the intervention group had significantly better outcomes for systolic blood pressure (SBP) [p $\leq 0.001]$, diastolic blood pressure (DBP) [p $\leq 0.001]$, physical functioning [ $p$ $\leq 0.001]$, role-physical $[p \leq 0.001]$, general health $[p=0.001]$, bodily pain [p $\leq 0.001]$, vitality [ $\leq 0.001]$, social functioning [ $p=0.027]$, role-emotional [ $p=0.003]$, and mental health $[p=0.001]$ compared to the control group. However, the differences in BMI between the groups were insignificant. Pan et al. concluded that tai chi is an effective therapy to improve SBP and DBP for patients suffering from essential hypertension. Zou et al. [151] found that the practice of baduanjin was beneficial for quality of life $(p=0.004)$, sleep quality $(p=0.001)$, balance $(p=0.004)$, handgrip strength $(p=0.007)$, trunk flexibility $(\mathrm{p}=0.006)$, systolic $(\mathrm{p}=0.0004)$ and diastolic $(\mathrm{p}$ $=0.005$ ) blood pressure, and resting heart rate $(p=0.0005)$. They examined the results of various studies on each of these topics. In the case of the effect of baduanjin on blood pressure, they examined 9 studies having a total of 743 participants. 
Ladawan et al. [152] investigated the effects of qigong exercise on cognitive function, blood pressure and cardiorespiratory fitness in 12 healthy middle-aged subjects who performed qigong exercises in 60-minute sessions, three times a week for eight weeks. They found that the exercises resulted in significant improvements in Trail Making Tests Part A ( $p=0.04)$, systolic blood pressure ( $p=0.0001)$, diastolic blood pressure $(p=0.005)$, mean arterial pressure $(p<0.001)$ and maximal workload $(p=0.032)$. Twelve weeks after cessation of the exercises, they had all returned to the baseline. The authors concluded that it is necessary to perform qigong regularly to maintain the improved health effects.

Ching et al. [153] examined data on 370 subjects from seven randomized controlled trials (RCTs). The following six types of qigong exercises were used:

\section{a) Conventional Qigong \\ b) Guolin Qigong \\ c) Shuxinpingxue Gong \\ d) Dongeui Qigong \\ e) Ba Duan Jin Qigong \\ f) Mawangdui Daoyinshu Qigong}

They found that the practice of qigong exercises had a significant effect on reducing systolic $(p<0.001)$ and diastolic $(p$ $<0.001$ ) blood pressure. The above studies are representative of the studies that have been done in recent years on the effectiveness of tai chi and qigong on reducing high blood pressure. Some other recent studies are listed in the reference section at the end of this article [154-188].

\section{Conflict of Interests}

None.

\section{References}

1. (2021) Wikipedia. Tai chi.

2. (2021) Wikipedia. Chen-style taijiquan.

3. (2021) Wikipedia. Yang-style t'ai chi ch'uan.

4. (2021) Wikipedia. Wu (Hao)-style t’ai chi ch'uan.

5. (2021) Wikipedia. Sun-style t'ai chi ch'uan.

6. Douglas Bill (1999) The Complete Idiot's Guide to T'ai Chi \& Qi Gong. New York: Alpha Books, a division of Macmillan General Reference.

7. Ross David-Dorian (2014) Essentials of Tai Chi and Qigong: Course Guidebook. Chantilly, VA: The Great Courses.

8. (2021) Wikipedia. History of Qigong.

9. Allen Katherine (2017) The Qigong Bible: The Definitive Guide to Energy Cultivation Exercise. Godsfield, UK.
10. Carson Patrick Emilio (2015) Liminality, Embodiment and the Six Healing Sounds of Qigong. Doctoral Dissertation. Florida State University.

11. Ce Jin, Hu Zhanggui, Jin Zhenghua (compilers) (1996) Practical Chinese Qigong for Home Health Care. Beijing: Foreign Languages Press.

12. Chang Pei-Shiun (2016) Physical and Psychological Effects of Qigong Exercise in Community-Dwelling Older Adults: An Exploratory Study. $\mathrm{PhD}$ dissertation. Yale University.

13. Chia Manta (1993) Healing Light of the Tao: Foundational Practices to Awaken Chi Energy. Rochester: Destiny Books.

14. Chia Mantak (2001) Cosmic Healing I: Cosmic Chi Kung. Chiang Mai, Thailand: Universal Tao Publications.

15. Chia Mantak, Dirk Oellibrandt (2001) Cosmic Healing II: Taoist Cosmology and Universal Healing Connections. Chiang Mai, Thailand: Universal Tao Publications.

16. Cohen Kenneth S (1999) The Way of Qigong: The Art and Science of Chinese Energy. Wellspring/Ballantine.

17. Davis Deborah (2008) Women's Qigong for Health \& Longevity. Boston \& London: Shambhala.

18. Downey, Robert G (2018) Qigong for Internal Growth: Eight Brocades and Other Exercises to Develop Your Energy. Golden Flower Internal Arts.

19. Frantzis Bruce (2005) Opening the Energy Gates of Your Body: Qigong for Lifelong Health. Blue Snake Books.

20. Frantzis Bruce (2010) Dragon and Tiger Medical Qigong, Volume 1. Fairfax, CA: Energy Arts.

21. Frantzis Bruce (2014) Dragon and Tiger Medical Qigong Volume 2: Qi Cultivation Principles and Exercises. North Atlantic Books.

22. Frantzis Bruce, Paul Cavel (2020) Heaven and Earth Qigong Volume One: Heal Your Body and Awaken Your Chi. Amazon.com.

23. Friedman Suzanne (2006) Medical Qigong Exercise Prescriptions: A Self-Healing Guide for Patients \& Practitioners. Xlibris.

24. Friedman Suzanne (2006) The Yijing Medical Qigong System: A Daoist Medical I-Ching Approach to Healing. Xlibris.

25. Friedman Suzanne B (2009) Heal Yourself with Qigong. New Harbinger Publications.

26. Jahnke Roger (1997) The Healer Within. San Francisco: Harper.

27. Jahnke Roger (2002) The Healing Promise of Qi. New York: Contemporary Books, a division of McGraw-Hill.

28. Jingwei li, Zhu Jianping (2014) The Illustrated Handbook of Chinese Qigong Forms from the Ancient Texts. London \& Philadelphia: Singing Dragon.

29. Johnson, Jerry Alan (2000) Chinese Medical Qigong Therapy: A Comprehensive Clinical Guide. Pacific Grove, CA: International Institute of Medical Qigong.

30. Johnson, Jerry Alan (2005) Chinese Medical Qigong Therapy, Vol. 1: Energetic Anatomy and Physiology. Pacific Grove, CA: International Institute of Medical Qigong.

31. Johnson, Jerry Alan (2005) Chinese Medical Qigong Therapy, Vol. 2: Pacific Grove, CA: International Institute of Medical Qigong.

32. Johnson Jerry Alan (2002) Chinese Medical Qigong Therapy, Vol. 3: Pacific Grove, CA: International Institute of Medical Qigong.

33. Johnson, Jerry Alan (2002) Chinese Medical Qigong Therapy, Vol. 4: Prescription Exercises and Meditations, Treatment of Internal Diseases, 
Pediatrics, Geriatrics, Gynecology, Neurology, and Energetic Psychology. Pacific Grove, CA: International Institute of Medical Qigong.

34. Johnson, Jerry Alan (2005) Chinese Medical Qigong Therapy, Vol. 5: An Energetic Approach to Oncology. Pacific Grove, CA: International Institute of Medical Qigong.

35. Kohn Livia (2008) Chinese Healing Exercises: The Tradition of Daoyin. University of Hawaii Press.

36. Korahais Anthony (2014) Help! I Can't Decide What Qigong Exercises to Practice! Blog. April 9.

37. Korahais Anthony (2017) 13 Proven Benefits of Qigong \& Tai Chi. FlowingZen.com.

38. Korahais Anthony (2017) History of Qigong: The 5 Categories of Q Cultivation. Bloc Post, February 14

39. Korahais Anthony (2017) Which Qigong Exercise Should You Practice for [Insert Problem]? Blog. October 10.

40. Korahais Anthony (2019) Do You Need Specific Qigong Exercises for Specific Problems? Blog. May 7.

41. Liang Shou-yu, Wenching wu (2010) Qigong Empowerment: A Guide to Medical and Wushu Energy Cultivation. East Providence, RI: Way of the Dragon Publishing.

42. McGee Robert W (2020) Qigong: A Bibliography of Books and Other Materials, Fayetteville State University, Broadwell College of Business and Economics, Studies in the Economics of Qigong, No. 1, August 25

43. McGee Robert W (2020) A Bibliography of Recent Medical Research on Qigong, Fayetteville State University, Broadwell College of Business and Economics, Studies in the Economics of Qigong, No. 2, August 31.

44. McGee Robert W (2020) Ba Duan Jin as a Treatment for Physica Ailments: A Bibliography of Recent Medical Research, Fayetteville State University, Broadwell College of Business and Economics, Studies in the Economics of Qigong, No. 3, August 31.

45. McGee Robert W (2020) Wu Qin Xi as a Treatment for Physical Ailments: A Bibliography of Recent Medical Research, Fayetteville State University, Broadwell College of Business and Economics, Studies in the Economics of Qigong, No. 4, August 31.

46. McGee, Robert W (2020) The Use of Yi Jin Jing to Treat Illness: A Summary of Three Studies, Fayetteville State University, Broadwell College of Business and Economics, Studies in the Economics of Qigong, No. 5, August 31

47. McGee Robert W (2020) Qigong and the Treatment and Prevention of COVID-19, Fayetteville State University, Broadwell College of Business and Economics, Studies in the Economics of Qigong, No. 6, September 4

48. McGee, Robert W (2020) Qigong and the Treatment and Prevention of Ca ncer, Fayetteville State University, Broadwell College of Business and Economics, Studies in the Economics of Qigong, No. 7, September 14.

49. McGee, Robert W (2021) Tai Chi, Qigong and Transgender Health Fayetteville State University, Broadwell College of Business and Economics, Studies in the Economics of Tai Chi and Qigong, No. 8, April 16

50. Murakawa Haruhiko (2002) Phenomenology of the Experience of Qigong: A Preliminary Research Design for the Intentional Bodily Practices. PhD dissertation. California Institute of Integral Studies.

51. Ramlal Derek (2007) Qigong and the Modernization of China. Doctoral Dissertation (D.A.), Saint John's University (New York).

52. Tapajna Mark (2006) An Examination of Medical Qigong: Its Practice, Application, and Potential Value for a Western Society. PhD dissertation. Cleveland State University.
53. (2021) Wikipedia. Qigong.

54. (2021) Wikipedia. 2021. History of Qigong.

55. (2021) Wikipedia. Baduanjin qigong.

56. (2021) Wikipedia. Liu Zi Jue.

57. (2021) Wikipedia. Five Animals.

58. (2021) Wikipedia. Yi Jin Jing.

59. Wu Zhongxian (2011) Chinese Shamanic Cosmic Orbit Qigong. London \& Philadelphia: Singing Dragon.

60. Wu Zhongxian (2012) Fire Dragon Meridian Qigong: Essential NeiGong for Health and Spiritual Transformation. London \& Philadelphia: Singing Dragon.

61. Wu Zhongxian (2012) Vital Breath of the Dao: Chinese Shamanic Tiger Qigong - Laohu Gong. London \& Philadelphia: Singing Dragon.

62. Yang Jwing-Ming (1997) The Root of Chinese Qigong: Secrets of Health, Longevity \& Enlightenment. Roslindale, MA: YMAA Publication Center.

63. Yang Jwing-Ming (1997) Eight Simple Qigong Exercises for Health: The Eight Pieces of Brocade. Roslindale, MA: YMAA Publication Center.

64. Yang Jwing-Ming (1998) Qigong for Health and Martial Arts: Exercises \& Meditation. Boston, MA: YMAA Publication Center

65. Yang Jwing-Ming (2000) Qigong: The Secret of Youth. Boston, MA: YMAA Publication Center.

66. Liu Zi Jue (2007) Chinese Health Qigong Association. Beijing: Foreign Languages Press.

67. Ba Duan Jin (2008) Chinese Health Qigong Association. Beijing: Foreign Languages Press.

68. Wu Qin Xi (2008) Chinese Health Qigong Association. Beijing: Foreign Languages Press.

69. Yi Jin Jing (2009) Chinese Health Qigong Association. Beijing: Foreign Languages Press.

70. Da Wu (2014) Chinese Health Qigong Association. Beijing: Foreign Languages Press.

71. Shi Er Duan Jin (2014) Chinese Health Qigong Association. Beijing: Foreign Languages Press.

72. Daoyin Yangsheng Gong Shi Er Fa (2014) Chinese Health Qigong Association. Beijing: Foreign Languages Press.

73. Mawanhdui Daoyin Shu (2014) Chinese Health Qigong Association. Beijing: Foreign Languages Press.

74. Taiji Yangsheng Zhang (2014) Chinese Health Qigong Association. Beijing: Foreign Languages Press.

75. Liu B, Fan Z, Wang Z, Li M, Lu T, et al. (2020) The efficacy and safety of Health Qigong for ankylosing spondylitis: Protocol for a systematic review and meta-analysis. Medicine 99(3): e18734.

76. Xie Y, Guo F, Lu Y, Guo Y, Wei G, et al. (2019) A 12-week Baduanjin Qigong exercise improves symptoms of ankylosing spondylitis: A randomized controlled trial. Complementary therapies in clinical practice, 36: 113119

77. Chou, Yvonne Wai Yi (2011) The Effects of Qigong on Reducing Stress, Anxiety and Enhancing Body-mind Wellbeing. PhD dissertation. Hong Kong Polytechnic University.

78. Lam, Kam Chuen (2014) The Qigong Workbook for Anxiety. Oakland, CA: New Harbinger Publications.

79. McGee, Robert W (2021) Tai Chi, Qigong and the Treatment of Depression and Anxiety. Biomedical Journal of Scientific \& Technical Research 36(2): 28350-28354 (2021). 
80. Liu Tianjun, Xiao Mei Qiang (2013) Chinese Medical Qigong. London \& Philadelphia: Singing Dragon.

81. Li Z, Liu S, Wang L, Smith L (2019) Mind-Body Exercise for Anxiety and Depression in COPD Patients: A Systematic Review and Meta-Analysis. International journal of environmental research and public health, 17(1): 22.

82. Liu F, Cui J, Liu X, Chen KW, Chen X, Li R, et al. (2020) The effect of tai chi and Qigong exercise on depression and anxiety of individuals with substance use disorders: a systematic review and meta-analysis. BMC complementary medicine and therapies 20(1): 161.

83. McGee, Robert W (2021) Tai Chi, Qigong and the Treatment of Arthritis. Biomedical Journal of Scientific \& Technical Research 37(5): 2972429734.

84. Yang Jwing-Ming (2005) Arthritis Relief: Chinese Qigong for Healing and Prevention. Roslindale, MA: YMAA Publication Center.

85. Yang Jwing-Ming (2017) Pain-Free Joints: Simple Qigong Movements for Arthritis Healing and Prevention. Roslindale, MA: YMAA Publication Center.

86. (2021) Tai Chi for Arthritis Video. Dr. Paul Lam. Free Lesson and Introduction. Tai Chi for Health Institute.

87. Ye J, Simpson MW, Liu Y, Lin W, Zhong W, et al. (2020) The Effects of Baduanjin Qigong on Postural Stability, Proprioception, and Symptoms of Patients with Knee Osteoarthritis: A Randomized Controlled Trial. Frontiers in medicine 6: 307 .

88. Ye J, Zheng Q, Zou L, Yu Q, Veronese N, et al. (2020) Mindful Exercise (Baduanjin) as an Adjuvant Treatment for Older Adults (60 Years Old and Over) of Knee Osteoarthritis: A Randomized Controlled Trial. Evidencebased complementary and alternative medicine: eCAM, 2020, 9869161.

89. Zeng ZP, Liu YB, Fang J, Liu Y, Luo J (2020) Effects of Baduanjin exercise for knee osteoarthritis: A systematic review and meta-analysis. Complementary therapies in medicine 48: 102279.

90. Rodrigues JM, Mestre M, Fredes LI (2019) Qigong in the treatment of children with autism spectrum disorder: A systematic review. J Integr Med 17(4): 250-260

91. Park J, Krause Parello CA, Barnes CM (2020) A Narrative Review of Movement-Based Mind-Body Interventions: Effects of Yoga, Tai Chi, and Qigong for Back Pain Patients. Holistic nursing practice 34(1): 3-23.

92. Zou L, Zhang Y, Yang L, Loprinzi PD, Yeung AS, et al. (2019) Are Mindful Exercises Safe and Beneficial for Treating Chronic Lower Back Pain? A Systematic Review and Meta-Analysis of Randomized Controlled Trials. Journal of clinical medicine 8(5): 628.

93. McGee, Robert W (2020) Qigong and the Treatment and Prevention of Cancer, Fayetteville State University, Broadwell College of Business and Economics, Studies in the Economics of Qigong, No. 7: 21.

94. McGee, Robert W (2021) Tai Chi, Qigong and the Treatment of Cancer. Biomedical Journal of Scientific \& Technical Research 34(5): 2717327182.

95. Sveund Jennifer (2017) The Experience of Qigong Among Women Cancer Survivors. Antioch University. Dissertations \& Theses. 378.

96. Arring NM, Barton DL, Brooks T, Zick SM (2019) Integrative Therapies for Cancer-Related Fatigue. Cancer journal (Sudbury, Mass.) 25(5): 349356.

97. Campbell KL, Zadravec K, Bland KA, Chesley E, Wolf F, et al. (2020) The Effect of Exercise on Cancer-Related Cognitive Impairment and Applications for Physical Therapy: Systematic Review of Randomized Controlled Trials. Physical therapy 100(3): 523-542.

98. Chang PS, Knobf T (2019) Qigong Exercise and Tai Chi in Cancer Care. Asia-Pacific journal of oncology nursing 6(4): 315-317.
99. Cheng TC, Lee YH, Mar CL, Huang WT, Chang YP (2020) The Health Promoting Mindfulness or Qigong Educational Programs for Beneficial Lifestyle Changes of Cancer Survivors. Journal of cancer education: the official journal of the American Association for Cancer Education 35(4): 743-750.

100. Deng G (2019) Integrative Medicine Therapies for Pain Management in Cancer Patients. Cancer journal (Sudbury, Mass.) 25(5): 343-348.

101. Ford CG, Vowles, KE, Smith BW, Kinney AY (2020) Mindfulness and Meditative Movement Interventions for Men Living with Cancer: A Meta-analysis. Annals of behavioral medicine: a publication of the Society of Behavioral Medicine 54(5): 360-373.

102. Garland SN, Mahon K, Irwin MR (2019) Integrative Approaches for Sleep Health in Cancer Survivors. Cancer journal (Sudbury, Mass.) 25(5): 337-342.

103. Larkey L, Kim W, James D, Kishida M, Vizcaino M, et al. (2020) MindBody and Psychosocial Interventions May Similarly Affect Heart Rate Variability Patterns in Cancer Recovery: Implications for a Mechanism of Symptom Improvement. Integrative cancer therapies 19: 1534735420949677

104. Li F, Wang C (2020) A Good Guy Again: Biosociality in a Cancer Selfhelp Organization. Medical anthropology: 1-14.

105. Lin WF, Zhong MF, Zhou QH, Zhang YR, Wang H, et al. (2019) Efficacy of complementary and integrative medicine on health-related quality of life in cancer patients: a systematic review and meta-analysis. Cancer management and research 11: 6663-6680.

106. Maindet C, Burnod A, Minello C, George B, Allano G, et al. (2019) Strategies of complementary and integrative therapies in cancerrelated pain-attaining exhaustive cancer pain management. Supportive care in cancer: official journal of the Multinational Association of Supportive Care in Cancer 27(8): 3119-3132.

107. Oberoi D, Piedalue KL, Pirbhai H, Guirguis S, Santa Mina D, et al. (2020) Factors related to dropout in integrative oncology clinical trials: interim analysis of an ongoing comparative effectiveness trial of mindfulness-based cancer recovery and Tai chi/Qigong for cancer health (The MATCH study). BMC research notes 13(1): 342.

108. Sowada KM (2019) Qigong: Benefits for Survivors Coping with CancerRelated Fatigue. Clinical journal of oncology nursing 23(5): 465-469.

109. Wang FF, Yuan Y, Song YJ, Wu YQ He Y, et al. (2020) Exercise or sport activities for patients with cancer: A protocol for overview of systematic reviews and meta-analyses. Medicine 99(20): e20084.

110. Kreutz C, Schmidt ME, Steindorf, K (2019) Effects of physical and mind-body exercise on sleep problems during and after breast cancer treatment: a systematic review and meta-analysis. Breast cancer research and treatment 176(1): 1-15.

111. Osypiuk K, Ligibel J, Giobbie Hurder A, Vergara Diaz G, Bonato P, et al. (2020) Qigong Mind-Body Exercise as a Biopsychosocial Therapy for Persistent Post-Surgical Pain in Breast Cancer: A Pilot Study. Integrative cancer therapies 19: 1534735419893766.

112. Zhang Q, Gao X, Liu S, Yu L, Zhu J, et al. (2020) Therapies for cognitive impairment in breast cancer survivors treated with chemotherapy: A protocol for systematic review. Medicine 99(19): e20092.

113. Henshall CL, Allin L, Aveyard H (2019) A Systematic Review and Narrative Synthesis to Explore the Effectiveness of Exercise-Based Interventions in Improving Fatigue, Dyspnea, and Depression in Lung Cancer Survivors. Cancer nursing 42(4): 295-306.

114. Kinney AY, Blair CK, Guest DD, Ani JK, Harding EM, et al. (2019) Biobehavioral effects of Tai Chi Qigong in men with prostate cancer: Study design of a three-arm randomized clinical trial. Contemporary clinical trials communications 16: 100431 
115. Zuniga KB, Chan JM, Ryan CJ, Kenfield SA (2020) Diet and lifestyle considerations for patients with prostate cancer. Urologic oncology 38(3): 105-117.

116. Ibañez GE, Fennie K, Larkey L (2019) Qigong as a promising mindbody exercise for cognitive functioning: Letter to Editor. Journal of the American Geriatrics Society 67(7): 1533-1534.

117. Wu C, Tang C (2019) Reply to: Qigong as a Promising Mind-Body Exercise for Cognitive Functioning. Journal of the American Geriatrics Society 67(7): 1534-1535.

118. Zou L, Loprinzi PD, Yeung AS, Zeng N, Huang T (2019) The Beneficial Effects of Mind-Body Exercises for People with Mild Cognitive Impairment: A Systematic Review with Meta-analysis. Archives of physical medicine and rehabilitation 100(8): 1556-1573.

119. Li Z, Liu S, Wang L, Smith L (2019) Mind-Body Exercise for Anxiety and Depression in COPD Patients: A Systematic Review and Meta-Analysis. International journal of environmental research and public health 17(1): 22 .

120. ChenJM, Wang ZY, Chen YJ, Ni J (2020) The Application of Eight-Segment Pulmonary Rehabilitation Exercise in People with Coronavirus Disease 2019. Frontiers in physiology 11: 646.

121. McGee Robert W (2020) Qigong and the Treatment and Prevention of COVID-19, Fayetteville State University, Broadwell College of Business and Economics, Studies in the Economics of Qigong No. 6: 10.

122. Zhang S, Zhu Q, Zhan C, Cheng W, Mingfang X, et al. (2020) Acupressure therapy and Liu Zi Jue Qigong for pulmonary function and quality of life in patients with severe novel coronavirus pneumonia (COVID-19): a study protocol for a randomized controlled trial. Trials 21(1): 751

123. Gaik Frances Veronica (2003) Merging East and West: A Preliminary Study Applying Spring Forest Qigong to Depression as an Alternative and Complementary Treatment. Psy.D. dissertation. Adler School of Professional Psychology.

124. McGee, Robert W (2021) Tai Chi, Qigong and the Treatment of Depression and Anxiety. Biomedical Journal of Scientific \& Technical Research 36(2): 28350-28354.

125. Gill BK, Cant R, Lam L, Cooper S, Lou V (2020) Non-pharmacological depression therapies for older Chinese adults: A systematic review \& meta-analysis. Archives of gerontology and geriatrics 88: 104037.

126. Gouw V, Jiang Y, Seah B, He H, Hong J, Wang W (2019) Effectiveness of internal Qigong on quality of life, depressive symptoms and selfefficacy among community-dwelling older adults with chronic disease: A systematic review and meta-analysis. International journal of nursing studies 99: 103378.

127. Henshall CL, Allin L, Aveyard H (2019) A Systematic Review and Narrative Synthesis to Explore the Effectiveness of Exercise-Based Interventions in Improving Fatigue, Dyspnea, and Depression in Lung Cancer Survivors. Cancer nursing 42(4): 295-306.

128. Li Z, Liu S, Wang L, Smith L (2019) Mind-Body Exercise for Anxiety and Depression in COPD Patients: A Systematic Review and Meta-Analysis. International journal of environmental research and public health 17(1): 22

129. Liu F, Cui J, Liu X, Chen KW, Chen X, et al. (2020) The effect of tai chi and Qigong exercise on depression and anxiety of individuals with substance use disorders: a systematic review and meta-analysis. BMC complementary medicine and therapies 20(1): 161.

130. Lu EY, Lee P, Cai S, So W, Ng B, et al. (2020) Qigong for the treatment of depressive symptoms: Preliminary evidence of neurobiological mechanisms. International journal of geriatric psychiatry 35(11): 1393-1401.
131. So WWY, Cai S, Yau SY, Tsang HWH (2019) The Neurophysiological and Psychological Mechanisms of Qigong as a Treatment for Depression: A Systematic Review and Meta-Analysis. Front Psychiatry 10: 820.

132. Zaprutko T, Göder R, Rybakowski F, Kus K, Kopciuch D, et al. (2020) Nonpharmacological treatments of inpatients with major depression - The case of Polish (Poznan) and German (Kiel) hospital. Complementary therapies in clinical practice 39: 101129.

133. Henshall CL, Allin L, Aveyard H (2019) A Systematic Review and Narrative Synthesis to Explore the Effectiveness of Exercise-Based Interventions in Improving Fatigue, Dyspnea, and Depression in Lung Cancer Survivors. Cancer nursing 42(4): 295-306.

134. De Sousa, Liliana Filipa Martins (2012) Effects of Qigong on the Balance of Elderly People: A Feasibility Study. Master Thesis of Traditional Chinese Medicine. Universidade do Porto, Portugal.

135. Li Zhi Cheng (2010) The Situation and Countermeasures of Health Qigong in Shanghai Older Age Groups of Community. Master's Degree Thesis. East China Normal University.

136. Lee P, Cai S, Lu EY, Ng B, Jensen MP, et al. (2020) Qigong Reduces Depressive Symptoms of Taiwanese Elderly with Chronic Physical Illness: A Randomized Controlled Trial. Journal of alternative and complementary medicine (New York, N.Y.) 26(1): 76-78.

137. Liu X, Seah J, Pang B, Tsao MA, Gu F, et al. (2020) A single-arm feasibility study of community-delivered Baduanjin (Qigong practice of the eight Brocades) training for frail older adults. Pilot and feasibility studies 6: 105.

138. Sarmento, Caio Vinicius Messias (2019) Changes in Immune Response to High Intensity Exercise in Patients Fibromyalgia before and after a Mind-Body Intervention. PhD dissertation. University of Kansas.

139. Sarmento C, Moon S, Pfeifer T, Smirnova IV, Colgrove Y, et al. (2020) The therapeutic efficacy of Qigong exercise on the main symptoms of fibromyalgia: A pilot randomized clinical trial. Integrative medicine research 9(4): 100416.

140. Fick Franklin (2018) Immortal Fang's Longevity Qigong: Rubbing the Abdomen to Prevent Illness and Prolong Life. Amazon.com.

141. Fick Franklin (2018) 24 Section Brocade Qigong: Strengthen Body Longevity Method. Amazon.com.

142. Lei Yan (2009) Instant Health: The Shaolin Qigong Workout for Longevity. Yan Lei Press.

143. Moon Sanghee, Caio VMS, Irina VS, Yvonne C, Kelly EL, et al. (2019) Effects of a Mind-Body Exercise on Non-motor Symptoms and Inflammatory Biomarkers in Parkinson's Disease. PhD dissertation. University of Kansas 6(1): 13.

144. Jin X, Wang L, Liu S, Zhu L, Loprinzi, PD, et al. (2019) The Impact of Mind-body Exercises on Motor Function, Depressive Symptoms, and Quality of Life in Parkinson's Disease: A Systematic Review and Metaanalysis. International journal of environmental research and public health 17(1): 31.

145. Yost Terri Lynn (2011) Qigong as a Novel Intervention in Service Members with Mild Traumatic Brain Industry. PhD dissertation. University of Virginia.

146. PubMed.gov https://pubmed.ncbi.nlm.nih.gov/

147. Liu D, Yi L, Sheng M, Wang G, Zou Y (2020) The Efficacy of Tai Chi and Qigong Exercises on Blood Pressure and Blood Levels of Nitric Oxide and Endothelin-1 in Patients with Essential Hypertension: A Systematic Review and Meta-Analysis of Randomized Controlled Trials. Evid Based Complement Alternat Med 2020: 3267971. 
148. Pan X, Tian L, Yang F, Sun J, Li X, et al. (2021) Tai Chi as a Therapy of Traditional Chinese Medicine on Reducing Blood Pressure: A Systematic Review of Randomized Controlled Trials. Evidence-Based Complementary and Alternative Medicine 2021: 4094325.

149. Zou L, SasaKi JE, Wang H, Xiao Z, Fang Q, et al. (2017) A Systematic Review and Meta-Analysis Baduanjin Qigong for Health Benefits: Randomized Controlled Trials. Evid Based Complement Alternat Med 2017: 4548706 .

150. Ladawan S, Klarod K, Philippe M, Menz V, Versen I, et al. (2017) Effect of Qigong exercise on cognitive function, blood pressure and cardiorespiratory fitness in healthy middle-aged subjects. Complement Ther Med 33: 39-45.

151. Ching SM, Mokshashri NR, Kannan MM, Lee KW, Sallahuddin NA, et al. (2021) Effects of qigong on systolic and diastolic blood pressure lowering: a systematic review with meta-analysis and trial sequential analysis. BMC Complement Med Ther 21(1): 8.

152. Carlson LE, Zelinski EL, Speca M, Balneaves LG, Jones JM, et al. (2017) Protocol for the MATCH study (Mindfulness and Tai Chi for cancer health): A preference-based multi-site randomized comparative effectiveness trial (CET) of Mindfulness-Based Cancer Recovery (MBCR) vs. Tai Chi/Qigong (TCQ) for cancer survivors. Contemp Clin Trials 59: 64-76.

153. Chen D (2016) Effect of Health Qigong Mawangdui Daoyinshu on Blood Pressure of Individuals with Essential Hypertension. J Am Geriatr Soc 64(7): 1513-1515.

154. Chen KW, Turner FD (2004) A case study of simultaneous recovery from multiple physical symptoms with medical qigong therapy. Altern Complement Med 10(1): 159-162.

155. Cheung BM, Lo JL, Fong DY, Chan MY, Wong SH, et al. (2005) Randomised controlled trial of qigong in the treatment of mild essential hypertension. J Hum Hypertens 19(9): 697-704.

156. Freeman SR, Hanik SA, Littlejohn ML, Malandruccolo AA, Coughlin J, et al. (2014) Sit, breathe, smile: effects of single and weekly seated Qigong on blood pressure and quality of life in long-term care. Complement Ther Clin Pract 20(1): 48-53.

157. Guo X, Zhou B, Nishimura T, Teramukai S, Fukushima M (2008) Clinical effect of qigong practice on essential hypertension: a meta-analysis of randomized controlled trials. J Altern Complement Med 14(1): 27-37.

158. Hartley L, Lee MS, Kwong JS, Flowers N, Todkill D, et al. (2015) Qigong for the primary prevention of cardiovascular disease. Cochrane Database Syst Rev 2015(6): CD010390.

159. Kuan SC, Chen KM, Wang C (2012) Effectiveness of Qigong in promoting the health of wheelchair-bound older adults in long-term care facilities. Biol Res Nurs 14(2): 139-146.

160. Kuang AK, Chen JL, Lu YR (1989) [Changes of the sex hormones in female type II diabetics, coronary heart disease, essential hypertension and its relations with kidney deficiency, cardiovascular complications and efficacy of traditional Chinese medicine or qigong treatment]. Zhong Xi Yi Jie He Za Zhi 9(6): 331-334, 323.

161. Lauche R, Peng W, Ferguson C, Cramer H, Frawley J, et al. (2017) Efficacy of Tai Chi and qigong for the prevention of stroke and stroke risk factors: A systematic review with meta-analysis. Medicine (Baltimore) 96(45): e8517.

162. Lee MS, Lee MS, Kim HJ, Moon SR (2003) Qigong reduced blood pressure and catecholamine levels of patients with essential hypertension. Int ] Neurosci 113(12): 1691-701.

163. Lee MS, Lee MS, Choi ES, Chung HT (2003) Effects of Qigong on blood pressure, blood pressure determinants and ventilatory function in middle-aged patients with essential hypertension. Am J Chin Med 31(3): 489-97.
164. Lee MS, Lee MS, Kim HJ, Choi ES (2004) Effects of qigong on blood pressure, high-density lipoprotein cholesterol and other lipid levels in essential hypertension patients. Int J Neurosci 114(7): 777-786.

165. Lee MS, Lim HJ, Lee MS (2004) Impact of qigong exercise on selfefficacy and other cognitive perceptual variables in patients with essential hypertension. J Altern Complement Med 10(4): 675-680.

166. Lee MS, Pittler MH, Guo R, Ernst E (2007) Qigong for hypertension: a systematic review of randomized clinical trials. J Hypertens 25(8): 1525-1532.

167. Leung KP, Yan T, Li LS (2001) Intracerebral haemorrhage and Qigong. Hong Kong Med J 7(3): 315-318.

168. Leung PC (2017) Rehabilitation training in artificially heated environment. J Exerc Rehabil 13(5): 546-549.

169. Liu X, Miller YD, Burton NW, Brown WJ (2010) A preliminary study of the effects of Tai Chi and Qigong medical exercise on indicators of metabolic syndrome, glycaemic control, health-related quality of life, and psychological health in adults with elevated blood glucose. $\mathrm{Br} \mathrm{J}$ Sports Med 44(10): 704-709.

170. Mayer M (1999) Qigong and hypertension: a critique of research. J Altern Complement Med 5(4): 371-382.

171. Nahas R (2008) Complementary and alternative medicine approaches to blood pressure reduction: An evidence-based review. Can Fam Physician 54(11): 1529-1533.

172. Niu JF, Zhao XF, Hu HT, Wang JJ, Liu YL, et al. (2019) Should acupuncture, biofeedback, massage, Qi gong, relaxation therapy, device-guided breathing, yoga and tai chi be used to reduce blood pressure? Recommendations based on high-quality systematic reviews. Complement Ther Med 42: 322-331.

173. Park JE, Hong S, Lee M, Park T, Kang K, et al. (2014) Randomized, controlled trial of qigong for treatment of prehypertension and mild essential hypertension. Altern Ther Health Med 20(4): 21-30.

174. Park JE, Kim JE, Jung S, Kim A, Park H, et al. (2017) The Effect of Dongeui Qigong for Prehypertension and Mild Essential Hypertension. Evid Based Complement Alternat Med 2017: 4274538.

175. Park JE, Yang CS, Jung SY, Kim AR, Lee M, et al. (2019) Exploring the effects of expert-led qigong and self-practice qigong on blood pressure. Integr Med Res 8(4): 271-272.

176. Payne P, Fiering S, Leiter JC, Zava DT, Crane Godreau MA (2017) Effectiveness of a Novel Qigong Meditative Movement Practice for Impaired Health in Flight Attendants Exposed to Second-Hand Cigarette Smoke. Front Hum Neurosci 11: 67.

177. Rogers CE, Larkey LK, Keller C (2009) A review of clinical trials of tai chi and qigong in older adults. West J Nurs Res 31(2): 245-79.

178. Shao BY, Zhang XT, Vernooij RWM, Lv QY, Hou YY, et al. (2020) The effectiveness of Baduanjin exercise for hypertension: a systematic review and meta-analysis of randomized controlled trials. BMC Complement Med Ther 20(1): 304.

179. So WWY, Cai S, Yau SY, Tsang HWH (2019) The Neurophysiological and Psychological Mechanisms of Qigong as a Treatment for Depression: A Systematic Review and Meta-Analysis. Front Psychiatry 10: 820.

180. Sousa CM, Goncalves M, Machado J, Efferth T, Greten T, et al. (2012) Effects of qigong on performance-related anxiety and physiological stress functions in transverse flute music schoolchildren: a feasibility study. Zhong Xi Yi Jie He Xue Bao 10(8): 858-865.

181. Xiao C, Yang Y, Zhuang Y (2016) Effect of Health Qigong Ba Duan Jin on Blood Pressure of Individuals with Essential Hypertension. J Am Geriatr Soc 64(1): 211-213. 
182. Xing ZH, Li W, Pi DR (1993) [Effect of qigong on blood pressure and life quality of essential hypertension patients]. Zhongguo Zhong Xi Yi Jie He Za Zhi 13(7): 413-414; 388-3889.

183. Xiong X, Wang P, Li X, Zhang Y (2015) Qigong for hypertension: a systematic review. Medicine (Baltimore) 94(1): e352.

184. Yang H, Wu X, Wang M (2017) The Effect of Three Different Meditation Exercises on Hypertension: A Network Meta-Analysis. Evid Based Complement Alternat Med 2017: 9784271.

185. Zheng G, Chen B, Fang Q Lin Q Tao J, et al. (2019) Baduanjin exercise intervention for community adults at risk of ischamic stroke: A randomized controlled trial. Sci Rep 9(1): 1240.

ISSN: 2574-1241

DOI: 10.26717/BJSTR.2021.39.006256

Robert W McGee. Biomed J Sci \& Tech Res

(C) (P) This work is licensed under Creative

Submission Link: https://biomedres.us/submit-manuscript.php
186. Zou L, Zhang Y, Sasaki JE, Yeung AS, Yang L, et al. (2019) Wuqinxi Qigong as an Alternative Exercise for Improving Risk Factors Associated with Metabolic Syndrome: A Meta-Analysis of Randomized Controlled Trials. Int J Environ Res Public Health 16(8): 1396.

187. Zhang Q, Gao X, Liu S, Yu L, Zhu J, et al. (2020) Therapies for cognitive impairment in breast cancer survivors treated with chemotherapy: A protocol for systematic review. Medicine 99(19): e20092.

188. Cocke Mariad (2015) Altered Recovery Process after Fatiguing Exercise and Potential Benefits of Qigong in Patients with Fibromyalgia. Master of Science Thesis. University of Kansas.

\begin{tabular}{ll} 
BIOMEDICAL & Assets of Publishing with us \\
RESEARCHES & - Global archiving of articles \\
\hline Immediate, unrestricted online access & - Rigorous Peer Review Process \\
& - Anthors Retain Copyrights
\end{tabular}

